\title{
Seroprevalencia de anticuerpos contra Neospora caninum en bovinos de crianza extensiva en tres distritos de Parinacochas, Ayacucho
}

\author{
Seroprevalence of antibodies against Neospora caninum in extensive cattle \\ farming in three districts of Parinacochas, Ayacucho
}

Angela Fernández Cevallos ${ }^{1}$, Siever Morales-Cauti ${ }^{1,2}$

\section{Resumen}

El objetivo del presente estudio fue determinar la seroprevalencia de anticuerpos contra Neospora caninum en ganado bovino de crianza extensiva en los distritos de Chumpi, Coracora y Pullo de la provincia de Parinacochas, Ayacucho. Se trabajó con 209 muestras de suero sanguíneo de bovinos mayores a cuatro meses de edad. Las muestras fueron analizadas mediante la técnica ELISA indirecta con kit IDEXX Neospora 2X Ab para detectar anticuerpos contra $N$. caninum. Se encontró una seroprevalencia corregida de $12.2 \pm 4.4 \%$ con asociación significativa $(\mathrm{p}<0.05)$ para la variable procedencia. El distrito de Coracora presentó la mayor prevalencia con $19.7 \pm 7.5 \%$.

Palabras clave: Neospora caninum, anticuerpos, bovino, seroprevalencia, aborto, serodiagnóstico

\section{Abstract}

The aim of this study was to determine the seroprevalence of antibodies against Neospora caninum in cattle under an extensive production system in the districts of Chumpi, Coracora and Pullo of the province of Parinacochas, Ayacucho. In total, 209

\footnotetext{
${ }^{1}$ Carrera de Medicina Veterinaria y Zootecnia, Universidad Científica del Sur, Lima, Perú

${ }^{2}$ E-mail: sieverm@hotmail.com
}

Recibido: 26 de noviembre de 2020

Aceptado para publicación: 20 de mayo de 2021

Publicado: 24 de agosto de 2021

CLos autores. Este artículo es publicado por la Rev Inv Vet Perú de la Facultad de Medicina Veterinaria, Universidad Nacional Mayor de San Marcos. Este es un artículo de acceso abierto, distribuido bajo los términos de la licencia Creative Commons Atribución 4.0 Internacional (CC BY 4.0) [https:// creativecommons.org/licenses/by/4.0/deed.es] que permite el uso, distribución y reproducción en cualquier medio, siempre que la obra original sea debidamente citada de su fuente original 
blood serum samples from bovines older than four months of age were collected. The samples were analyzed using the indirect ELISA technique with the IDEXX Neospora 2X Ab kit to detect antibodies against $N$. caninum. A corrected seroprevalence of $12.2 \pm$ $4.4 \%$ was found with a significant association $(\mathrm{p}<0.05)$ for the origin variable. The district of Coracora presented the highest prevalence with $19.7 \pm 7.5 \%$.

Key words: Neospora caninum, antibodies, bovine, seroprevalence, abortion, serodiagnosis

\section{INTRODUCCIÓN}

El Perú cuenta con aproximadamente 5.2 millones de cabeza de ganado bovino, siendo el criollo el grupo racial predominante con $63.9 \%$ de la población (INEI, 2012). La población ganadera presenta una mayor distribución poblacional en la sierra, especialmente el ganado de doble propósito bajo un sistema de crianza de tipo extensivo (MINAGRI, 2017; Rosemberg, 2018).

Entre las enfermedades que afectan al ganado se encuentran parásitos como Neospora caninum que ocasiona principalmente abortos (Rivera, 2001; Dubey et al., 2007). Este parásito es un protozoario intracelular obligado del Phylum Apicomplexa (Khan et al., 2019), y se encuentra ampliamente distribuido (Khan et al., 2019; Ribeiro et al., 2019; Pereyra et al., 2020). El parásito posee un ciclo de vida heteroxeno facultativo y tiene como hospederos definitivos a los perros y a los coyotes (McAllister et al., 1998; Gondim et al., 2004; Wilson et al., 2016) у como hospederos intermediarios a una amplia gama de animales, entre los que destacan los bovinos por su mayor susceptibilidad (Moore et al., 2005; Dubey et al., 2006; Ribeiro et al., 2019). La parasitosis se adquiere por la ingestión de ooquistes excretados por los canes y transmitidos horizontalmente por vía digestiva a los bovinos (transmisión horizontal) (Girata, 2016), pero también puede transmitirse al feto vía transplacentaria (transmisión vertical); siendo ésta última vía considerada una de las principales fuentes de transmisión y persistencia de la infección en el bovino, ya que hembras infectadas perpetúan el parasitismo de generación en generación (Tuemmers et al., 2017; De Aquino et al., 2019).

La enfermedad se caracteriza por ser típicamente asintomática, con excepción del aborto en los bovinos (Dubey et al., 2017). La presentación ocurre a partir del segundo tercio de gestación, pudiendo nacer el ternero infectado y sin lesiones o asintomático (Martínez et al., 2012; Serrano et al., 2018); sin embargo, existe la posibilidad de la muerte del feto en el útero, nacimientos prematuros, neonatos débiles con problemas neuromusculares, momificación fetal, placentitis, disentería y fiebre aguda por dos o tres días (Dubey et al., 2007; Girata, 2016; Wilson et al., 2016). En el Perú se reportan prevalencias de $N$. caninum desde $3 \%$ hasta casi el 60\% (Atoccsa et al., 2005; SENASA, 2011; Vélez et al., 2013; Granados et al., 2014; Portocarrero et al., 2015; Arauco, 2018; Serrano et al., 2018).

Existen diversos métodos para identificar la presencia o infección de $N$. caninum, siendo estas técnicas histopatológicas, moleculares y de aislamiento (Dubey, 2003). Las pruebas inmunodiagnósticas disponibles son la inmunofluorescencia indirecta (IFI), ELISA, aglutinación directa, inmunohistoquímica (IHQ) y electroforesis combinada con inmunodetección (Western Immunblot) (Dubey, 1999). A nivel mundial, no existen protocolos estandarizados para el diagnóstico de neosporosis bovina (Dubey et al., 2007; 
McAllister, 2016); sin embargo, el método común de diagnóstico en los estudios seroepidemiológicos es mediante la técnica de ELISA indirecta que permite la identificación de anticuerpos en el suero sanguíneo de los animales (Álvarez, 2003; Da Silva et al., 2019). Esta técnica es confiable debido a que posee una sensibilidad y especificidad mayor a las obtenidas con la IFI; además, carece de subjetividad y permite procesar un gran número de muestras (Rodríguez, 2015; Serrano et al., 2018). Por todo ello, el presente estudio buscó determinar la frecuencia de seroreactores a anticuerpos contra Neospora caninum en bovinos de crianza extensiva en tres distritos de la provincia de Parinacochas, Perú.

\section{Materiales y Métodos}

El presente estudio se desarrolló en mayo de 2018, comprendiendo los distritos de Coracora, Chumpi y Pullo de la provincia de Parinacochas, ubicada al sur de Ayacucho (Perú), a una altitud entre 2800 hasta 3500 $\mathrm{msnm}$. La zona posee planicies, colinas y laderas de montañas, con temperaturas que oscilan entre los $6.6^{\circ} \mathrm{C}$ hasta $\operatorname{los} 25.5^{\circ} \mathrm{C}$ (Aronés et al., 2003; SENAMHI, 2019). El procesamiento de las muestras se realizó en el Laboratorio de Microbiología de la Universidad Científica del Sur, Lima.

El muestreo fue de tipo aleatorio, considerando animales mayores a cuatro meses de edad, sin distinción de sexo, raza, o categoría zootécnica (Rojas, 2010). Además, se entrevistó a los propietarios de cada fundo para identificar el estado reproductivo y edad de los bovinos muestreados.

El tamaño mínimo de muestra se calculó mediante la fórmula para estimar poblaciones finitas (Wayne, 2012), considerando la población bovina de los distritos de Coracora (13 988 bovinos), Chumpi (5339) y de Pullo (7736), dando un total de 27063 bo- vinos (INEI, 2012). Se utilizó una seroprevalencia referencial de $15.01 \%$ (SENASA, 2011), con un nivel de confianza de $95 \%$ y un error admisible de $5 \%$. Esto dio como resultado un tamaño mínimo de muestra de 197 animales; que fueron distribuidos proporcionalmente en Coracora $(n=102)$, Chumpi (n=39) y Pullo ( $n=57)$. No obstante, se llegaron a muestrear 209 animales.

Las muestras de sangre fueron colectadas en tubos de $10 \mathrm{ml}$ al vacío sin anticoagulante por punción directa en la vena yugular. Las muestras fueron transportadas al laboratorio del hospital central de la ciudad de Coracora dentro de las cuatro horas siguientes y fueron centrifugadas a $1600 \mathrm{G}$ por 5 minutos. El suero resultante fue almacenado a $-20{ }^{\circ} \mathrm{C}$ hasta su procesamiento.

Las muestras fueron procesadas mediante la técnica de ELISA indirecta utilizando el kit Neospora X2 Ab (IDEXX) para confirmar la presencia de anticuerpos contra $N$. caninum. Los resultados de seroprevalencia se presentaron en porcentajes de frecuencia con intervalo de confianza al 95\% (Wayne, 2012). Los resultados son presentados, asimismo, mediante la caja de bigotes. Se determinó, además, la seroprevalencia corregida considerando los índices de sensibilidad (98.5\%) y especificidad (97.9\%) del kit empleado (Álvarez-García et al., 2013). El análisis estadístico se realizó mediante la prueba de Chi-cuadrado para determinar la posible asociación entre la presencia de anticuerpos contra $N$. caninum y las variables sexo, categoría zootécnica, raza, procedencia y estado reproductivo.

\section{Resultados}

Se detectaron anticuerpos contra $N$. caninum en 29 animales de los 209 muestreados, obteniendo una seroprevalencia cruda de $13.9 \%\left(\mathrm{IC}_{95 \%}: 9.2-18.3 \%\right)$ y una prevalencia corregida de $12.2 \%\left(\mathrm{IC}_{95 \%}: 7.8\right.$ - 
Cuadro 1. Seroprevalencia de Neospora caninum $(\mathrm{n}=209)$ en bovinos de crianza extensiva de los distritos de Chumpi, Coracora y Pullo, Ayacucho

\begin{tabular}{|c|c|c|c|c|c|c|c|c|}
\hline \multirow{2}{*}{ Variables } & \multirow{2}{*}{ Positivos } & \multirow{2}{*}{ Total } & \multirow{2}{*}{$\begin{array}{c}\text { Prevalencia } \\
\text { cruda } \\
(\%)\end{array}$} & \multicolumn{2}{|c|}{$\begin{array}{c}\text { Intervalo de } \\
\text { confianza 95\% }\end{array}$} & \multirow{2}{*}{$\begin{array}{c}\text { Prevalencia } \\
\text { real } \\
(\%) \\
\end{array}$} & \multicolumn{2}{|c|}{$\begin{array}{c}\text { Intervalo de } \\
\text { confianza 95\% }\end{array}$} \\
\hline & & & & Mínimo & Máximo & & Mínimo & Máximo \\
\hline \multicolumn{9}{|l|}{ Sexo } \\
\hline Macho & 5 & 24 & 20.8 & 4.6 & 37.1 & 19.4 & 3.6 & 35.26 \\
\hline Hembra & 24 & 185 & 13.0 & 8.1 & 17.8 & 11.3 & 6.72 & $\begin{array}{c}15.84 \\
p=0.295\end{array}$ \\
\hline \multicolumn{9}{|c|}{ Categoría zootécnica } \\
\hline Ternero & 1 & 5 & 20.0 & 0.0 & 55.1 & 18.5 & 0.0 & 52.66 \\
\hline Vaquilla & 1 & 17 & 5.9 & 0.0 & 17.1 & 3.9 & 0.0 & 13.15 \\
\hline Torete & 3 & 16 & 18.8 & 0.0 & 37.9 & 17.3 & 0.0 & 35.79 \\
\hline Vaca & 23 & 156 & 14.7 & 9.2 & 20.3 & 13.1 & 0.0 & 18.42 \\
\hline Toro & 1 & 6 & 16.7 & 0.0 & 46.5 & 15.1 & 0.0 & 43.77 \\
\hline Vaquillona & 0 & 9 & 0 & 0 & 5.0 & 0 & 0 & $\begin{array}{c}0 \\
\mathrm{p}=0.704\end{array}$ \\
\hline \multicolumn{9}{|l|}{ Raza } \\
\hline Criollo & 17 & 120 & 14.2 & 7.9 & 20.4 & 12.5 & 6.6 & 18.44 \\
\hline Holstein & 0 & 6 & 0.0 & 0.0 & 0.0 & 0 & 0 & 0 \\
\hline B. Swiss & 12 & 68 & 17.6 & 8.6 & 26.7 & 16.1 & 7.39 & 24.87 \\
\hline Simmental & 0 & 8 & 0.0 & 0.0 & 5.0 & 0 & 0 & 0 \\
\hline Hereford & 0 & 7 & 0 & 0 & 5.0 & 0 & 0 & $\begin{array}{c}0 \\
\mathrm{p}=0.379\end{array}$ \\
\hline \multicolumn{9}{|l|}{ Procedencia } \\
\hline Chumpi & 2 & 40 & 5.0 & 0.0 & 11.8 & 3.0 & 0 & 8.31 \\
\hline Coracora & 23 & 109 & 21.1 & 13.4 & 28.8 & 19.7 & 12.24 & 27.18 \\
\hline Pullo & 4 & 60 & 6.7 & 0.4 & 13.0 & 4.7 & 0 & $\begin{array}{c}10.12 \\
p=0.007\end{array}$ \\
\hline \multicolumn{9}{|c|}{ Estado reproductivo } \\
\hline Vacía & 20 & 140 & 14.3 & 8.5 & 20.1 & 13.2 & 7.59 & 18.81 \\
\hline Preñada & 3 & 16 & 18.8 & 0.0 & 37.9 & 17.3 & 0 & $\begin{array}{c}35.79 \\
p=0.633\end{array}$ \\
\hline Total & 29 & 209 & 13.9 & 9.2 & 18.6 & 12.21 & 7.77 & 16.65 \\
\hline
\end{tabular}

16.6\%) (Cuadro 1). La menor seroprevalencia se observó en Chumpi (3.81\%) y la mayor en Coracora $(19.71 \%)(p<0.05)$. No se encontraron diferencias significativas para las variables raza, sexo, categorías zootécnicas ni estado reproductivo.
El diagrama de cajas y bigotes de la dispersión de la edad (Figura 1) presenta una mayor dispersión en la edad en los bovinos que resultaron negativos a la prueba. La dispersión de los resultados según el número de partos presentó varios valores atípicos en los 
animales con resultados negativos a la prueba diagnóstica (Figura 2).

\section{Discusión}

La seroprevalencia cruda de $13.9 \%$ con una prevalencia corregida de $12.21 \%$ (Cuadro 1) resultó similar al $15.0 \%$ reportado por SENASA (2012) en la misma región de Ayacucho, pero inferior a otras reportadas en diversas regiones de Perú, como en Oxapampa con $18.8 \%$ (Portocarrero et al., 2015), Lima con 46.6\% (Serrano et al., 2018), Tacna con $28.7 \%$ (Vélez et al., 2013) y Junín con $15.3 \%$ (Arauco, 2018). Prevalencias variadas han sido reportadas en otros países de América del Sur; así, Venezuela 21\% (Pinilla y Da Silva, 2018), Chile 21.1\% (Tuemmers et al., 2017), Ecuador 23.4\% (Maldonado et al., 2020), Argentina 35.3\% (Pereyra et al., 2020), Paraguay 41.4\% (Gimenez et al., 2019), Colombia 52\% (Cruz et al., 2019) y Brasil 71.4\% (De Aquino et al., 2019), diferencias que podrían ser atribuidas al sistema de producción (Serrano et al., 2018; Ribeiro et al., 2019), programas de sanidad (Ribeiro et al., 2019) y susceptibilidad racial (Dubey et al., 2007). Por otro lado, las características climáticas influyen en la viabilidad de los ooquistes (Gharekhani y Mohammad, 2019).

El lugar de procedencia fue la única variable que presentó asociación estadísticamente significativa $(\mathrm{p}<0.05)$ con la prevalencia de anticuerpos contra $N$. caninum, teniendo la localidad de Coracora la mayor seroprevalencia con un $19.71 \pm 7.47 \%$, posiblemente por las condiciones de crianza y mayor presencia de canes lo cual es considerado como un factor de riesgo de importancia, debido a su potencial contribución a la transmisión horizontal (Vanleeuwen et al., 2010; Ribeiro et al., 2019; Pereyra et al., 2020), posibilitando mayor contacto con material placentario eliminado, permitiendo la transmisión horizontal debido a la ingestión de ooquistes. Otra característica del distrito de Coracora es que presentan hatos con un mayor número de animales, favoreciendo potencialmente una mayor oportunidad de transmisión del patógeno. Se dispone de reportes que mencionan que predios con más de 100 bovinos tienen un mayor riesgo de infección (Portocarrero et al., 2015; Gharekhani y Mohammad, 2019).

El sexo del animal no tuvo asociación estadística con la ocurrencia de neosporosis (Cuadro 1), coincidiendo con otros estudios (Vega, 2010; Gharekhani y Mohammad, 2016, 2019; Satragno et al., 2019); no obstante, se indica que las diferencias hormonales (estro y segundo trimestre de la gestación) podrían cumplir un rol importante en la susceptibilidad del huésped a la infección por $N$. caninum (López et al., 2005; Dubey et al., 2007). Por otro lado, la variable raza no presentó asociación significativa con respecto a la seropositividad frente a $N$. caninum; coincidiendo con Munhoz et al. (2009), habiendo más una relación con el sistema de producción (Moore et al., 2005; Dubey et al., 2007), debido al mayor tiempo de vida de los animales en el sistema de producción lechero.

No se estableció asociación estadística significativa entre la edad y el diagnóstico de anticuerpos contra $N$. caninum, tal y como se observa en la literatura (Vélez et al., 2013; Cruz et al., 2019, Ribeiro et al., 2019). Sin embargo, otras investigaciones reportan la edad como un factor de riesgo asociado a la neosporosis bovina, dado que a mayor edad existe un mayor riesgo de seropositividad (Atoccsa et al., 2005; Pullido et al., 2016; Banegas, 2018; Olmo et al., 2019; Semango et al., 2019). Además, Dijkstra et al. (2001) sugiere la importancia de incluir animales jóvenes a los estudios, ya que los terneros con infección congénita tienen altos niveles de anticuerpos.

Para el presente estudio se seleccionaron los tres distritos con mayor número de ganado. El trabajo se realizó en condiciones de crianza extensiva, donde la falta de registros, plan sanitario e información de los sistemas de producción no permiten concluir de 


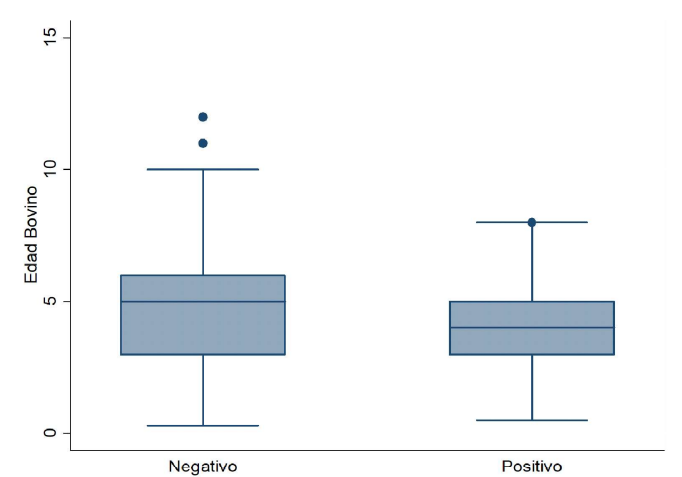

Figura 1. Diagrama de dispersión de la edad de 209 bovinos de Ayacucho, según el resultado a la prueba diagnóstica de Neospora caninum

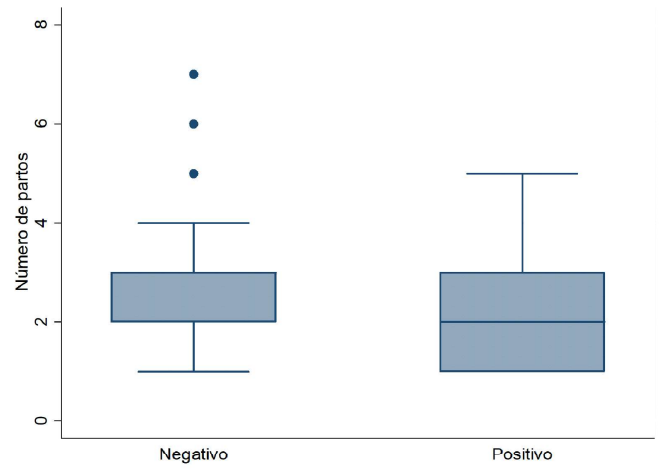

Figura 2. Diagrama de caja y bigotes de la frecuencia de bovinos positivos y negativos a la prueba diagnóstica de $N$. caninum según el número de partos

forma categórica con la identificación de factores de riesgos o características propias de la infección producido por el patógeno en estudio. Sin embargo, a pesar de las limitaciones, los resultados obtenidos son importantes para el entendimiento de la epidemiología y útil para poder implementar un plan de manejo, prevención y control de la enfermedad. De otra parte, cabe recalcar que los datos recolectados confirman la presencia del parásito, más no la enfermedad.

\section{Conclusiones}

- La seroprevalencia corregida de anticuerpos contra Neospora caninum es de $12.2 \pm 4.4 \%$, confirmando su presencia en los distritos de Chumpi, Coracora y Pullo de la provincia de Parinacochas, Ayacucho en 2018.

- Existe asociación estadística significativa $(p<0.05)$ entre los distritos de Chumpi, Coracora y Pullo con la presencia de anticuerpos contra el parásito Neospora caninum.

\section{Literatura Citada}

1. Álvarez G, Collantes E, Costas E, Rebordosa X, Ortega L. 2003. Influence of age and purpose for testing on the cut-off selection of serological methods in bovine neosporosis. Vet Res 34: 341-352. doi: 10.1051/vetres:2003009

2. Alvarez G, García A, Gutiérrez D, Navarro V, Pastor I, Ortega L. 2013. Serological diagnosis of bovine neosporosis: A comparative study of commercially available ELISA tests. Vet Parasitol 198: 85-95. doi: 10.1016/ j.vetpar.2013.07.033

3. Arauco F. 2018. Seroprevalencia y factores de riesgo de neosporosis bovina en el valle del Mantaro-Región Junín, Perú. Rev Inv Vet Perú 29: 1430-1439 doi: 10.15381/rivep.v29i4.15195

4. Aronés M, Apel K, Caro I, Castillo L, Hurtado L, Quinteros E, Vargas $C$. 2003. Centralismo y descentralización en Ayacucho. [Internet]. Disponible en: https://www.academia.edu/3367764/ UN BREVE PERFIL DE PARINACOCHAS

5. Atoccsa J, Chávez A, Casas E, Falcón N. 2005. Seroprevalencia de Neospora caninum en bovinos lecheros criados al pastoreo en la provincia de Melgar-Puno. Rev Inv Vet Perú 16: 70-75. doi: 10.15381/rivep.v16i1.1541 
6. Banegas D. 2018. Seroprevalencia de Neospora caninum en vacunos Brown Swiss en el distrito de Caracoto, Puno. Tesis de Médico Veterinario Zootecnista. Puno, Perú: Univ. Nacional del Antiplano. $83 \mathrm{p}$.

7. Cruz S, Díaz A, Bulla D, García D, Pulido M. 2019. Diagnóstico serológico de Neospora caninum en vacas del municipio de Tuta, Bocayá. Rev Med Vet 66: 197-207. doi: 10.15446/rfmvz.v66n3.84256

8. Da Silva C, Armendado J, Moore D, Cantón G, Macías M, Riet F. 2019. Comparación de kits comerciales para la detección de anticuerpos en la investigación diagnostica del aborto asociado a Neospora caninum en rodeos lecheros de Uruguay. Rev Argent Microbiol 52: 107-114. doi: 10.1016/j.ram.-2019.06.004

9. De Aquino L, Minutti A, De Souza B, Ramos L, Melo M, Martins de Almeida B, Garcia J, De Barros L. 2019. Vertical transmission of Neospora caninum in bovine fetuses from a slaughterhouse in Brazil. Trop Anim Health Pro 51: 1751-1755. doi: 10.1007/s11250-01901828-y

10. Dijkstra T, Barkema HW, Eysker M, Beiboer ML, Wouda W. 2003. Evaluation of a single serological screening of dairy herds for Neospora caninum antibodies. Vet Parasitol 110: 161-169

11. Dubey J. 1999. Recent advances in Neospora and neosporosis. Vet Parasitol 84: 349-367. doi: 10.1016/s03044017(99)00044-8.

12. Dubey J. 2003. Review of Neospora caninum and neoporosis in animals. Korean J Parasitol 41:1-16. doi: 10.3347/ kjp.2003.41.1.1

13. Dubey J, Buxton D, Wouda W. 2006. Pathogenesis of bovine neosporosis. J Comp Pathol 134: 267-289. doi: 10.1016/ j.jcpa.2005.11.004
14. Dubey J, Schares G, Ortega L. 2007. Epidemiology and control of neosporosis and Neospora caninum. Clin Microbiol Rev 20: 323-367. doi: 10.1128/CMR.00031-06

15. Dubey J, Hemphill A, Calero R, Schares G. 2017. Neosporosis in animals. CRC Press. 548 p.

16. Gharekhani J, Mohammad Y. 2019. Neospora caninum infection in dairy farms with history of abortion in West of Iran. Vet Anim Sci 8: 100071. doi: 10.1016/j.vas.2019.10007

17. Gimenez G, Alonso N, Vera G, Pedrozo R. 2019. Seroprevalencia de la neosporosis y su efecto sobre el intervalo entre partos en vacas lecheras del departamento central, Paraguay - Año 2018. Compend Cienc Vet 9: 30-34. doi: 10.18004/compend.cienc.-vet.2019.09.01.30-34

18. Girata J. 2016. Estudio zootécnico de la neoporosis bovina: análisis teórico de orientación para los ganaderos de Santander y Boyacá. Tesis de Ingeniero Zootecnista. Bucaramanga, Colombia: Univ. Nac. Abierta y a Distancia. 76 p.

19. Gondim L, McAllister M, Pitt W, Zemlicka D. 2004. Coyotes (Canis latrans) are definitive hosts of Neospora caninum. Int J Parasitol 34: 159-161. doi: 10.1016/j.ijpara.2004.01.001

20. Granados S, Rivera H, Casas E, Suárez F, Arana C, Chávez A. 2014. Seroprevalencia de Neospora caninum en bovinos lecheros de cuatro distritos del Valle del Mantaro, Junín. Rev Inv Vet Perú 25: 58-64. doi: 10.15381/rivep.v25i1.8468

21. [INEI] Instituto Nacional de Estadística e Informática. 2012. IV Censo Nacional Agropecuario: Existencia de ganado, aves, otros animales y colmenas. Lima. Perú: [Internet]. Disponible en: http://censos.inei.gob.pe/cenagro/tabulados/ 
22. Khan A, Fujita A, Randle N, RegidorCerrillo J, Shaik J, Shen K, et al. 2019. Global selective sweep of a highly inbred genome of the cattle parasite Neospora caninum. P Natl Acad Sci USA 116: 22764-22773. doi: 10.1073/ pnas. 1920070116

23. López F, García I, Santolaria P, Yániz L, López M, Nogareda C, Almeria $S$. 2005. Relationship between rainfall and Neospora caninum - associated abortion in two dairy herds in dry enviromment. J Vet Med B 52: 112-118. doi: 10.1111/j.1439-0450.2005.00833.x

24. Maldonado J, Vallecillo A, Pérez C, Cirone K, Dorsch M, Morrell E, Scioli $V$, et al. 2020. Bovine neosporosis in dairy cattle from the southern highlands of Ecuador. Vet Parasitol 20: 100377. doi: 10.1016/j.vprsr.2020.100377

25. Martínez A, Moreno G, Cruz A. 2012. Actualización de la neosporosis bovina. Conexión Agropecuaria JDC 2: 49-66.

26. McAllister M, Dubey J, Lindsay D, Jolley W, Wills R, McGuire A. 1998. Dogs are definitive hosts of Neospora caninum. Inter J Parasitol 28: 14731478. doi: 10.1016/S0020-7519(98)00138-6

27. McAllister M. 2016. Diagnosis and control of bovine neosporosis. Vet Clin $\mathrm{N}$ Am-Food A 32: 443-463. doi: 10.1016/ j.cvfa.2016.01.012

28. [MINAGRI]. Ministerio de Agricultura y Riego. 2017. Plan Nacional de Desarrollo Ganadero 2017-2021. [Internet]. Disponible en: https://www.minagri.gob.pe/portal/download/pdf/especiales/ plan-nacional-ganadero.pdf

29. Moore D, Odeon A, Venturini M, Campero C. 2005. Neosporosis bovina: conceptos generales, inmunidad y perspectivas para la vacunación. Rev Argent Microbiol 37: 217-228.

30. Munhoz A, Pereira M, Flausino W. 2009. Neospora caninum seropositivity in cattle breeds in the South Fluminense
Paraíba valley, state of Rio de Janeiro. Pesqui Vet Brasil 29: 29-32. doi: 10.1590/ S0100-736X2009000100004

31. Olmo L, Reichel M, Nampanya S, Khounsy S, Wahl Ll, Clark B, Thomson P, et al. 2019. Risk factors for Neospora caninum, bovine viral diarrhoea virus, and Lepstospira interrogans serovar Hardjo infection in smallholder cattle and buffalo in Lao PDR. Plos One 14: e0220335, doi: 10.1371/journal.pone.-0220335

32. Pereyra W, Suarez V, Cardoso N, Gual I, Martínez G, Capozzo A, Mansilla F. 2020. Prevalencia sérica de Neospora caninum y factores de riesgo asociado a su transmisión en tambos de la provincia de Salta, Argentina. Rev Argent Microbiol 2020. doi: 10.1016/j.ram.2020.06.011

33. Pinilla, J, Da Silva N. 2018. Neospora caninum en bovinos doble propósito en fincas del estado Guárico, Venezuela. Rev Mex Cienc Pecu 9: 833-844. doi: 10.22319/rmcp.v9i4.4546

34. Portocarrero C, Pinedo R, Falcón N, Chávez A. 2015. Factores de riesgo asociadas a la seroprevalencia de Neospora caninum en bovinos naturalmente infectados en la ceja de selva de Oxapampa. Perú. Rev Inv Vet Perú 26: 119-126. doi: 10.15381/rivep.v26i1.10916

35. Pullido M, García D, Vargas J. 2016. Seroprevalencia de Neospora caninum en un hato lechero de Bocayá, Colombia. Rev Inv Vet Perú 27: 355-362. doi: 10.15381/rivep.v27i2.11658

36. Ribeiro C, Soares I, Mendes R, De Santis Bastos P, Katagiri S, Zavilenski R, de Abreu H, Afreixo V. 2019. Metaanalysis of the prevalence and risk factors associated with bovine neosporosis. Trop Anim Health Pro 51: 17831800. doi: 10.1007/s11250-019-01929-8

37. Rivera H. 2001. Causas frecuentes de aborto bovino. Rev Inv Vet Perú 12: 117-122. 
38. Rodríguez A. 2015. Transmisión horizontal y vertical de Neospora caninum en tres sistemas de cría bovina. Tesis de Maestría. Argentina: Univ. Nacional de Mar del Plata. $98 \mathrm{p}$.

39. Rojas M. 2010. Manejo de enfermedades en el ganado de carne y leche. Bogotá, Colombia: Uniminuto. $108 \mathrm{p}$.

40. Rosemberg M. 2018. La ganadería bovina en Perú. [Internet]. Disponible en: http://www.veterinariadigital.com/ articulos/la-ganaderia-bovina-en-peru

41. Satragno D, Pavón-Rocha A, RábagoCastro J, De la Cruz-Hernández N. 2020. Alta seroprevalencia de Neospora caninum en perros con sospecha clínica de neosporosis en Montevideo. Rev Argent Microbiol 52: 165-166. doi: 10.1016/j.ram.2019.03.007

42. Semango G, Hamilton $C$, Kreppel $K$, Katzer F, Kibona T, Lankester F, Allan $\boldsymbol{K}$, et al. 2019. The sero-epidemiology of Neospora caninum in cattle in Northern Tanzania. Front Vet Sci 6: 327. doi: 10.3389 /fvets.2019.00327

43. [SENAMHI] Servicio Nacional de Meteorología e hidrología. 2019. [Internet]. Disponible en: https://www.senamhi.gob.pe/

44. [SENASA] Servicio Nacional de Sanidad Agraria. 2011. Proyecto: Fortalecimiento del sistema de vigilancia zoosanitario. Informe final: Caracterización de la diarrea viral bovina, neosporosis bovina y rinotraqueitis infecciosa bovina en el Perú. Lima, Perú: SENASA. $26 \mathrm{p}$.

45. Serrano E, Evaristo R, Quispe M, Hinostroza E. 2018. Seroprevalencia de Neospora caninum en bovinos de Lima y comparación entre ELISA e IFI. Rev Inv Vet Perú 29: 916-922. doi: 10.15381/ rivep.v29i3.14757

46. Tuemmers $C$, Valenzuela $G$, Nuñez $C$, De la Cruz R, Meyer J, Andaur M, Leyan P, Mora C. 2017. Seroprevalencia de Neospora caninum en bovinos de una feria ganadera de la región de la Araucanía, Chile. Rev Inv Vet Perú 28: 629-635. doi: 10.15381/rivep.v28i3.12680

47. Vanleeuwen J, Haddad J, Dohoo I, Keefe G, Tiwari A, Scott H. 2010. Risk factors associated with Neospora caninum seropositivity in randomly sampled Canadian dairy cows and herds. Prev Vet Med 93: 129-138. doi: 10.1016/ j.prevetmed.2009.11.013

48. Vega L, Chávez A, Falcón N, Casas E, Puray N. 2010. Prevalencia de Neospora caninum en perros pastores de una empresa ganadera de la sierra sur de Perú. Rev Inv Vet Perú 21: 80-86.

49. Vélez V, Cahuana J, Pérez O. 2013. Seroprevalencia de Neospora caninum en bovinos lecheros en el sector Sama Grande del Distrito de Sama-Inclán, Tacna - Perú. Veritas 14: 100-104

50. Wayne D. 2012. Bioestadística base para el análisis de las ciencias de la salud. $4^{\mathrm{a}}$ ed. México DF: Limusa. 915 p.

51. Wilson D, Orsel K, Waddington J, Rajeev M, Sweeny A, Joseph T, Raverty S. 2016. Neospora caninum is the leading cause of bovine fetal loss in British Columbia, Canada. Vet Parasitol 218: 46-51. doi: 10.1016/ j.vetpar.2016.01.006 